\title{
Acute pancreatitis in a 61-year-old man with COVID-19
}

\author{
Shay Brikman MD, Veronika Denysova MD, Husam Menzal MD, Guy Dori MD PhD
}

Cite as: CMAJ 2020 July 27;192:E858-9. doi: 10.1503/cmaj.201029

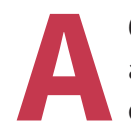

61-year-old previously healthy man who was not taking any medications was admitted to hospital with fever, dyspnea and cough, which he had for 5 days. Our patient had been in close contact with his father, who had confirmed coronavirus disease 2019 (COVID-19). Radiography of his chest showed bilateral opacities compatible with viral pneumonia, and results from testing of nasal and throat swabs using real-time transcription polymerase chain reaction assay were positive for severe acute respiratory syndrome coronavirus 2 (SARS-CoV-2).

Within 3 days of our patient's admission to hospital, severe COVID-19 pneumonia developed, ${ }^{1}$ with tachypnea and hypoxemia that required high-flow oxygen supplementation, fever and weight loss. We initially prescribed azithromycin (500 mg daily for $3 \mathrm{~d}$ ), hydroxychloroquine (loading dose of $800 \mathrm{mg}$ on day 1 , followed by $200 \mathrm{mg}$ twice daily for $10 \mathrm{~d}$ ) and zinc (50 mg twice daily for $5 \mathrm{~d}$ ). In addition, he received acetaminophen as needed (1000 mg on 4 different occasions). We later prescribed tocilizumab (anti-interleukin-6 receptor antibody, 2 doses of $400 \mathrm{mg}$ administered intravenously), dexamethasone (10 mg twice daily administered intravenously for $5 \mathrm{~d}$ with tapering thereafter), enoxaparin (80 $\mathrm{mg}$ twice daily for $10 \mathrm{~d}$ ), lopinavir/ritonavir (2 tablets of $200 \mathrm{mg} / 50 \mathrm{mg}$ twice daily for $5 \mathrm{~d}$ ) and pantoprazole (40 mg daily administered intravenously). A culture of his sputum identified Staphylococcus aureus, and we added ciprofloxacin (400 mg twice daily administered intravenously) and clindamycin (300 mg administered orally 4 times a day for $7 \mathrm{~d}$ ). Seven days later, our patient's respiratory status had improved, and he no longer required high-flow oxygen.

On day 14 , our patient reported sudden diffuse abdominal pain with anorexia. He was afebrile and had no vomiting or diarrhea. His abdomen was soft, with mild diffuse tenderness but no signs of peritoneal irritation. A complete blood cell count showed his level of leukocytes was elevated at 37 (normal 4.5-11.5) $\times 10^{9} / \mathrm{L}$ with 93\% neutrophils, and he had an absolute lymphocyte count of 0.98 (normal $1.5-5.0) \times 10^{9} / \mathrm{L}$. His serum lipase level was elevated at 203 (normal 21-67) U/L, and he had a serum amylase level of $142 \mathrm{U} / \mathrm{L}$ (28-100 U/L). His levels of alkaline phosphatase and direct bilirubin were in the normal range. Contrast-enhanced computed tomography (CT) of our patient's abdomen showed signs of pancreatitis (Figure 1). ${ }^{2}$ We diagnosed acute pancreatitis and started supportive treatment, including nothing by mouth, intravenous fluids and analgesia.

Two days later, our patient's gastrointestinal symptoms had resolved completely, and all abnormal laboratory values had

\section{KEY POINTS}

- Acute pancreatitis has been associated with severe coronavirus disease 2019 (COVID-19).

- Medications used to manage severe COVID-19 have been associated with acute pancreatitis.

- Unresolved abdominal pain occurring late in the course of COVID-19 warrants a thorough work-up, which may include testing for levels of amylase, lipase, liver enzymes and triglycerides, and abdominal computed tomography.

returned to normal. We ran additional blood tests to investigate the cause of acute pancreatitis. ${ }^{3}$ His levels of liver enzymes and calcium were normal. His serum level of triglycerides was mildly elevated $(3.18$ [normal 1.8 ] mmol/L; his level was normal $[<1.69 \mathrm{mmol} / \mathrm{L}] 1 \mathrm{~d}$ before the onset of abdominal pain and was $3.46 \mathrm{mmol} / \mathrm{L}$ on the day after the contrast-enhanced CT). Imaging did not show gallstones, or abnormalities of the gallbladder or bile duct. Our patient did not have a medical history of pancreatitis, and he had no history of heavy consumption of alcohol.

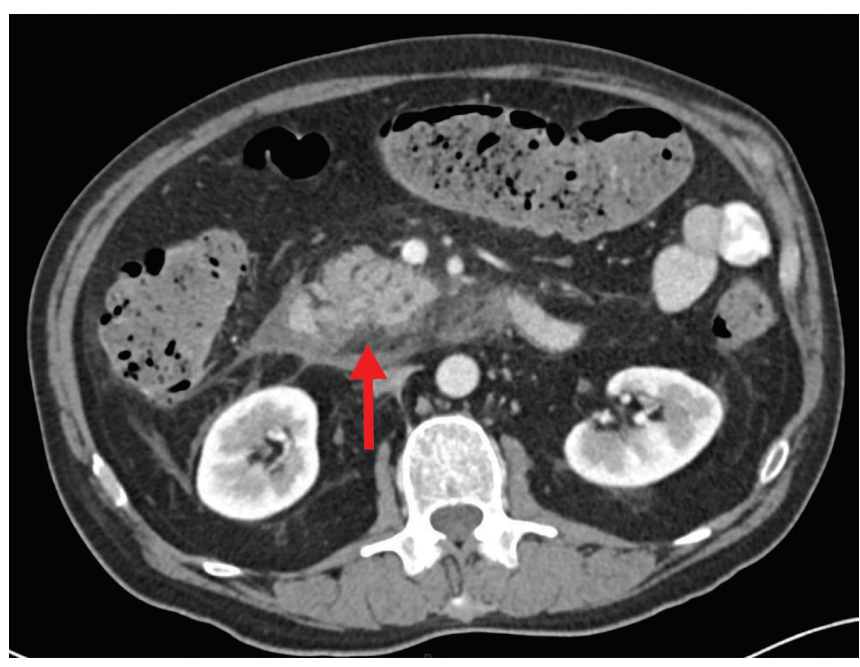

Figure 1: Abdominal contrast-enhanced computed tomography in a 61 -year-old man recovering from severe coronavirus disease 2019 pneumonia that shows signs of acute pancreatitis, including focal parenchymal enhancement of the pancreas head with inflammatory changes in peripancreatic fat (red arrow). 


\section{Discussion}

Acute pancreatitis is an inflammatory disorder of the pancreas, most commonly caused by gallstones and heavy alcohol consumption. ${ }^{4}$ The revised Atlanta classification system defines acute pancreatitis if at least 2 of the following 3 criteria are met in a patient: abdominal pain (acute onset of persistent and severe epigastric pain); increased serum lipase (or amylase) levels to greater than 3 times the upper limit of normal value; or characteristic findings of acute pancreatitis on contrast enhanced CT. ${ }^{2}$ Our patient met 2 out of 3 criteria, as his elevated lipase level did not exceed 3 times the upper limit of normal value. We propose that our patient's acute pancreatitis was most likely related to infection with SARS-CoV-2, medication used to manage the infection or a combination of both.

Up to $10 \%$ of acute pancreatitis is thought to have an infectious cause (mostly viral; e.g., mumps, coxsackievirus or cytomegalovirus) either through direct viral injury or through an immune-mediated inflammatory response. ${ }^{5}$ The SARS-CoV-2 Spike protein binds angiotensin-converting enzyme 2 (ACE2) protein of the host cell membrane to fuse into the cell for viral replication. Angiotensin-converting enzyme-2 is highly expressed on lung alveolar epithelial cells; ${ }^{6}$ however, overexpression of ACE2 in the pancreas has been reported recently. ${ }^{7}$

Our patient's presentation and onset of symptoms is consistent with previous findings that $17 \%$ of patients with severe COVID-19 have pancreatic injury with elevated serum amylase and lipase, and as many as $7 \%$ of patients have imaging findings compatible with pancreatitis. ${ }^{7,8} \mathrm{~A}$ case report involving $3 \mathrm{mem}$ bers of the same family who were admitted to hospital in Denmark found 2 cases of acute pancreatitis associated with COVID19 , both shortly after admission and with plasma levels of amylase over $900 \mathrm{U} / \mathrm{L}^{9}{ }^{9}$ In a letter to the editor of the British Journal of Surgery, Anand and colleagues reported a case involving a 59-year-old woman with COVID-19 in the United Kingdom who had acute pancreatitis 10 days after admission to hospital, which was complicated by bacterial pneumonia. She was treated with intravenous vancomycin and a course of doxycycline. ${ }^{10}$

Drug-related acute pancreatitis is relatively uncommon, accounting for less than $5 \%$ of all cases of acute pancreatitis, with numerous drugs being suggested as causative agents. ${ }^{3,4}$ During the course of our patient's disease, he received a combination of at least 4 drugs that have been associated with acute pancreatitis (i.e., acetaminophen, dexamethasone, ciprofloxacin and pantoprazole), ${ }^{3}$ in addition to tocilizumab, a known causative agent. ${ }^{11}$ Two recent reports described patients with COVID19 who developed pancreatitis with hypertriglyceridemia (level of serum triglyceride $>11.3 \mathrm{mmol} / \mathrm{L}$ ) during treatment with a combination of tocilizumab and lopinavir/ritonavir. ${ }^{12,13}$

Although case reports cannot establish causality, our patient's disease trajectory suggests that the manifestations of COVID-19 may include acute pancreatitis. Physicians should consider this possibility early or late in the course of COVID-19 illness.

In addition, using personal protective equipment may compromise the reliability of the physical examination of patients with COVID-19. Thus, unresolved abdominal pain warrants a thorough work-up, including testing for levels of amylase, lipase liver enzymes and triglycerides, and a complete blood cell count as an initial screening. If laboratory results are outside of normal ranges, clinicians may wish to consider further investigation with contrast-enhanced CT.

\section{References}

1. Wu Z, McGoogan JM. Characteristics of and important lessons from the coronavirus disease 2019 (COVID-19) outbreak in China: summary of a report of 72314 cases from the Chinese Center for Disease Control and Prevention. JAMA 2020 Feb. 24 [Epub ahead of print]. doi: 10.1001/jama.2020.2648.

2. Banks PA, Bollen TL, Dervenis C, et al.; Acute Pancreatitis Classification Working Group. Classification of acute pancreatitis - 2012: revision of the Atlanta classification and definitions by international consensus. Gut 2013;62:102-11.

3. Wolfe D, Kanji S, Yazdi F, et al. Drug induced pancreatitis: a systematic review of case reports to determine potential drug associations. PLoS One 2020; 15:e0231883.

4. Forsmark CE, Vege SS, Wilcox CM. Acute pancreatitis. N Engl J Med 2016;375: 1972-81.

5. Rawla P, Bandaru SS, Vellipuram AR. Review of infectious etiology of acute pancreatitis. Gastroenterology Res 2017;10:153-8.

6. Bourgonje AR, Abdulle AE, Timens W, et al. Angiotensin-converting enzyme-2 (ACE2), SARS-CoV-2 and pathophysiology of coronavirus disease 2019 (COVID-19). J Pathol 2020 May 20 [Epub ahead of print]. doi:10.1002/path.5471.

7. Liu F, Long X, Zhang B, et al. ACE2 expression in pancreas may cause pancreatic damage after SARS-CoV-2 infection. Clin Gastroenterol Hepatol 2020 Apr. 22 [Epub ahead of print]. S1542-3565(20)30537-1. doi:10.1016/j.cgh.2020.04.040.

8. Wang F, Wang H, Fan J, et al. Pancreatic injury patterns in patients with COVID-19 pneumonia. Gastroenterology 2020 Apr. 1 [Epub ahead of print]. S0016-5085 (20)30409-1. doi:10.1053/j.gastro.2020.03.055.

9. Hadi A, Werge M, Kristiansen KT, et al. Coronavirus disease-19 (COVID-19) associated with severe acute pancreatitis: case report on three family members. Pancreatology 2020;20:665-7.

10. Anand ER, Major C, Pickering O, et al. Acute pancreatitis in a COVID-19 patient Br J Surg 2020;107:e182.

11. Flaig $\mathrm{T}$, Douros A, Bronder E, et al. Tocilizumab-induced pancreatitis: case report and review of data from the FDA Adverse Event Reporting System. J Clin Pharm Ther 2016;41:718-21.

12. Rubel AR, Chong PL, Abdullah MS, et al. Lipemic serum in patients with (coronavirus disease 2019) COVID-19 undergoing treatment. J Med Virol 2020 Apr. 28 [Epub ahead of print]. doi: 10.1002/jmv.25942.

13. Morrison AR, Johnson JM, Ramesh M, et al. Acute hypertriglyceridemia in patients with COVID-19 receiving tocilizumab. J Med Virol 2020 Apr. 21 [Epub ahead of print]. doi: 10.1002/jmv.25907.

\section{Competing interests: None declared.}

This article has been peer reviewed.

The authors have obtained patient consent.

Affiliations: Departments of Internal Medicine E (Corona A) (Brikman, Denysova, Dori) and Surgery B (Menzal), HaEmek Medical Center, Afula, Israel; Faculty of Medicine (Brikman, Dori), Technion Israel Institute of Technology, Haifa, Israel

Contributors: Shay Brikman conceived the work and wrote the draft. Veronika Denysova, Husam Menzal and Guy Dori analyzed and interpreted the data and revised the manuscript critically for important intellectual content. All of the authors gave final approval of the version to be published and agreed to be accountable for all aspects of the work in ensuring that questions related to the accuracy or integrity of any part of the work are appropriately investigated and resolved.

Correspondence to: Shay Brikman, sbrikman@gmail.com 